\title{
Recurrence of Aggressive Urothelial Carcinoma in an Ileal Neobladder without Involvement of Remaining Urothelium
}

\author{
Amanda Fox ${ }^{*}$, Anna Crow ${ }^{2}$, Sara Khammash ${ }^{3}$, Mirna Knight ${ }^{4}$, Shandra Wilson ${ }^{1}$ \\ ${ }^{1}$ Centura Medical Group Urology, Centura Health, Greenwood Village, CO, USA \\ ${ }^{2}$ Department of Eberly College of Science, The Pennsylvania State University, State College, PA, USA \\ ${ }^{3}$ Department of Biology, University of Colorado Colorado Springs, Colorado Springs, CO, USA \\ ${ }^{4}$ Department of Pathology, Littleton Adventist Hospital, Littleton, CO, USA
}

*Correspondence to: Amanda M. Fox, CCMA, Centura Medical Group Urology, Centura Health, Greenwood Village, CO, USA; Tel: 3036493707; Fax: 3036493711; E-mail: amandafox@centura.org

Received: Jun 16, 2021 ; Accepted: Jun 20 ${ }^{\text {th }}, 2021$; Published: Jun 23 ${ }^{\text {rd }}, 2021$

Citation: Fox AM, Crow A, Khammash S, Knight M, Wilson S. Recurrence of aggressive urothelial carcinoma in an ileal neobladder without involvement of remaining urothelium. Urology Open A Open J. 2021; 2(2): 70-72. doi: 10.33169/uro.UOAOJ-2-119

\begin{abstract}
We review a case of a 70-year-old male with a recurrence of urothelial carcinoma in an ileal neobladder without involvement of the remaining urothelium two years after a radical cystoprostatectomy. This case is extremely rare especially due to the lack of recurrence in the urethra or upper urinary tract.
\end{abstract}

\section{Abbreviations}

BCG: Bacillus Calmette-Guérin; CIS: Carcinoma in situ; CT: Computerized/computed tomography; NCCN: National Comprehensive Cancer Network; PE: Pulmonary embolism; PET: Positron emission tomography; RC: Radical cystectomy; TURBT: Trans-urethral resection of bladder tumor; UC: Urothelial carcinoma.

Keywords: Urothelial carcinoma; Ileal; neobladder; Tumor; Recurrence; Metastasis.

\section{INTRODUCTION}

An orthotopic ileal neobladder is a widely used method of treatment after a radical cystectomy for invasive bladder cancer. Recurrences of urothelial carcinoma are not uncommon in the urethra or the upper urinary tract (0.75-6\% of cystectomy patients), ${ }^{1}$ but isolated recurrences of urothelial carcinoma in theileal neobladder are extremely rare. Although a recurrence in an orthotopic diversion has been reported in four other patients, this case is unique. This report outlines recurrence of urothelial carcinoma in an ileal neobladder two years after radical cystoprostatectomy wherein a patient had undergone radiation to the area of the neobladder prior, with no concurrent upper tract disease. Until this report, radiation to the neobladder had not been examined as a risk factor for a neobladder recurrence, and recurrences in diversions had only been described as a late phenomenon, occurring six years or more after cys- tectomy. $^{2-5}$

\section{CASE REPORT}

In November 2017, a 66-year-old male was found to have a bladder tumor while undergoing ureteroscopy with laser lithotripsy for treatment of nephrolithiasis. A TURBT was performed and pathology showed high-grade focally-invasive urothelial carcinoma (pT1) and carcinoma in situ (CIS). He returned for second-look TURBT one week later and was found to have residual CIS. The patient underwent repeat TURBT one month later and pathology showed non-invasive high-grade papillary urothelial carcinoma (pTa). After allowing time for his hematuria to resolve, the patient completed BCG induction treatment. The induction course was completed but was difficult due to hemorrhagic cystitis, PE when taken off of anticoagulation, and recurrent infection resulting 
in sepsis.

Following BCG induction, the patient underwent surveillance cystoscopy and TURBT. This revealed a recurrence of invasive urothelial carcinoma (pT1). He did not complete additional BCG treatments due to complications and side effects during the first round.

Six months later, during a surveillance cystoscopy, he was found to have another recurrence, and the tumor was resected. Pathology identified progression to muscle-invasive urothelial carcinoma (pT2). Four cycles of neoadjuvant gemcitabine and cisplatin were administered prior to radical cystoprostatectomy, lymph node dissection, and creation of ileal neobladder. The neoadjuvant chemotherapy did not successfully downstage and the final pathology showed invasive highgrade urothelial carcinoma of the bladder (pT3a) (Figure 1), two out of twenty-three lymph nodes were positive for urothelial carcinoma $(\mathrm{pN} 3)$ (Figure 2), a background of carcinoma in situ (CIS), and Gleason 3+3 acinar adenocarcinoma (pT2 pN0). Margins were read as negative. Due to the unfavorable pathology, the patient then began one year of adjuvant nivolumab immunotherapy.

Figure 1. Bladder pathology from RC showing invasion into muscularis propria at $40 \mathrm{x}$ magnification (left) and 200x magnification (right).

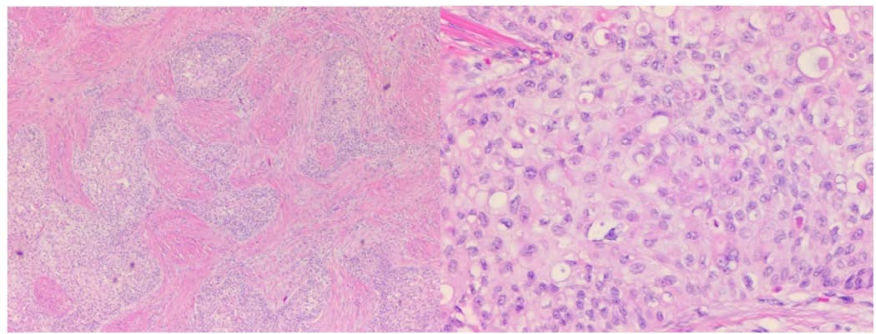

Figure 2. Lymph node showing metastatic carcinoma at 40x magnification (left) and lymphovascular invasion, 100x magnification (right).

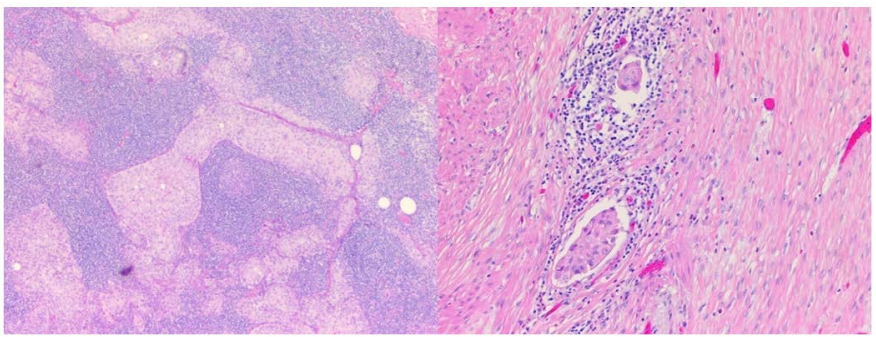

A follow-up PET scan in June 2019 showed pubic bone metastasis, so one month of targeted radiation therapy was completed simultaneously during the course of immunotherapy. The patient was free of recurrences for about one year, then a pelvic mass biopsy was performed after a suspicious surveillance CT scan and pathology showed a urothelial carcinoma metastasis. In December 2020, a CT scan revealed two liver metastases, retroperitoneal adenopathy, and a neobladder mass. In-office cystoscopy was performed and a suspicious neobladder mass was visualized. The mass appeared to resemble urothelial carcinoma. The patient was then scheduled for a TURBT of the neobladder mass, which was completely resected in February 2021. Pathology showed high-grade urothelial carcinoma of the neobladder that had extensive lymphovascular invasion and extensive invasion into the neobladder muscle and submucosa (Figures 3 and 4). No recurrence was seen in the urethra or the upper urinary tract at this time. There were no targetable mutations in the neobladder pathology. The patient is still alive, and follows up with regular surveillance cystoscopy and continued systemic therapy with his oncology team.

Figure 3. TURBT pathology showing urothelial carcinoma invading muscle of neobladder at $40 \mathrm{x}$ magnification (left) and 100x magnification (right).

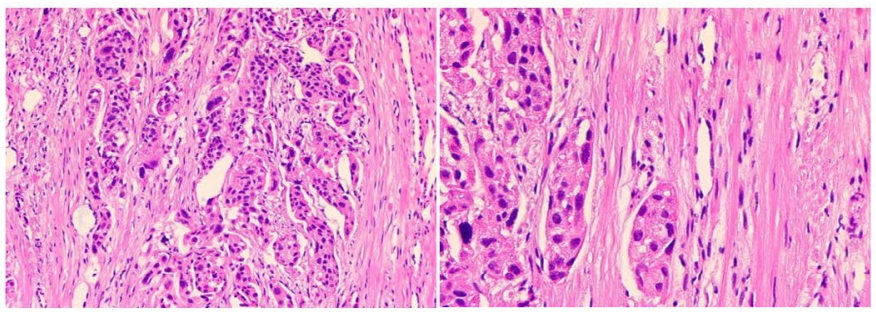

Figure 4. TURBT pathology showing the muscle wall of the neobladder with significant muscle invasion at 20x magnification (left), neobladder tissue with lymphovascular invasion at 40x magnification (middle), and neobladder wall showing involvement by recurrent carcinoma at 20x magnification (right).

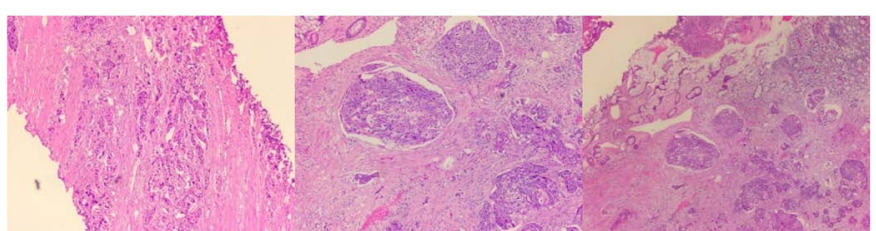

\section{DISCUSSION}

Recurrence of urothelial carcinoma after radical cystectomy is not uncommon..$^{1-6}$ Recurrence rates have been seen to be lower if a patient is treated with neoadjuvant micrometastatic-targeting chemotherapy. ${ }^{6,7}$ Recurrence rates increase with higher stages of disease on cystectomy and with nodal involvement. ${ }^{6,8}$ his patient had pT3a pN3 disease and was therefore at higher risk for recurrence.

The urethra is the most common location of recurrence of UC (0.7-18\% recurrence rate), however, our patient did not have a recurrence in this location. ${ }^{7,8}$

Pelvic soft tissue recurrences ( $45 \%$ recurrence rate) and metastatic lymphadenopathy (33\% recurrence rate) are two of the more common sites of recurrence and were both found in this patient. ${ }^{9}$ Less common are liver metastases (19\% recurrence rate) and bone metastases (12\% recurrence rate), while liver metastases are most often found in patients that had nodal involvement. ${ }^{9}$ Our patient had both bone and liver metastases prior to retroperitoneal nodal involvement.

Recurrences of UC within the ileal neobladder have been well documented, but cases that do not also involve any of the remaining urothelium are exceedingly rare, with only four cases being reported as of March 2019., ${ }^{2,5,9,10}$ Until this case, this has been seen as early as six years and as late as eleven years following RC. , $5,9,10$ Our patient's tumor occurred only two years after RC. It is well-known that all parts of the revised urinary system are at risk for recurrence of $\mathrm{UC}$, and many have tried to posit an explanation for how this process occurs. ${ }^{4}$ Two possible explanations are field change, or field cancerization, and seeding theory, or implantation theory. ${ }^{4,9,11}$ The seeding or implantation theory states that urothelial tumor cells are able to implant themselves in uro- 
thelial tissue in the bladder, the neobladder, and tissues other than the urothelium. ${ }^{911}$ More specifically, intraluminal tumor cell seeding is considered to be a potential factor for the causation of recurrences within tissues aside from the urothelium. ${ }^{4}$ In the case of our patient, the ileal tissue should not have a field cancerization effect and with no upper tract disease, direct seeding would be rare as well. A more likely mechanism stems from residual lymphatic disease that was not completely eradicated by surgery and chemotherapy and that reseeded into the neobladder. It is also possible that the lymphatics of the ileum were already involved at the time of surgery.

Another potential difference in our case compared to the other four reported cases is the fact that the patient had pelvic radiation after the creation of his ileal orthotopic diversion. This begs the question if radiation might be a risk factor for metaplasia and the transformation to cancer.

The figure below compares the 2019 RC pathology of the UC in the native bladder to the 2021 TURBT pathology of the UC recurrence in the ileal neobladder (Figure 5). Both slides are visually similar, showing that the neobladder tumor was indeed a urothelial carcinoma.

Figure 5. 2019 pathology from RC (left) and 2021 pathology from TURBT of neobladder (right) showing nearly identical histology.

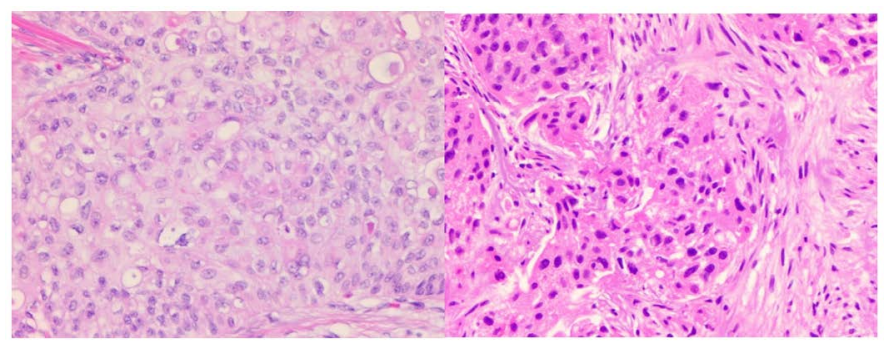

\section{CONCLUSION}

In this study, we report the case of a 70-year-old male who had a recurrence of urothelial carcinoma in an ileal neobladder without involvement of the remaining urothelium two years after a radical cystoprostatectomy and postoperative radiation. This case reminds us that radiologists should be aware that patients who had a radical cystoprostatectomy with orthotopic ileal neobladder should be monitored for recurrences of urothelial carcinoma in the neobladder if there is extensive disease in other non-urothelial tissues. [9] Although recurrences of muscle-invasive bladder cancer typically develop within 2 years of a radical cystectomy [10], cases of tumor recurrence have been reported up to 12 years following a radical cystectomy. [2] This suggests patient's likely benefit from regular long-term follow-up with their physicians and reminds providers to work up hematuria after cystectomy - not just to rule out disease of remaining urothelial tissue, but to rule out recurrence in the diversion as well.

\section{CONFLICTS OF INTEREST}

\section{REFERENCES}

1. Naser-Tavakolian A, Ghodoussipour S, DjaladatHooman. Upper Urinary Tract Recurrence Following Bladder Cancer Therapy: A Review of Surveillance and Management. Curr Opin Urol. 2019; 29(3): 189-197. doi: 10.1097/mou.0000000000000598

2. Doshi, C. P., Barkan, G. A., \& Quek, M. L. (2019). Urothelial Carcinoma Recurrence in an Orthotopic Neobladder without Urethral or Upper Urinary Tract Involvement. Case Reports in Urology. 2019; 1-3. doi: $10.1155 / 2019 / 8458706$

3. Yamashita R, Matsuzaki M, Niwakawa M, Ito I. Bacillus CalmetteGuérin Treatment of Urothelial Carcinoma Arising in The Ileal Neobladder After Radical Cystectomy. Int J Urol. 2014; 21(3): 333-334. doi: 10.1111/iju. 12268

4. Cakmak O, Tarhan H, Celik O, Kucuk U, Ilbey YO. Transitional Cell Carcinoma in Orthotopic Ileal Neobladder. Case Reports in Urology. 2014; 2-14: 1-3. doi: 10.1155/2014/218615

5. Cherbanyk F, Prod'homme M, Pezzetta, E, Chevaux B, Anaye A, Boillat JJ. (2016). Urothelial Carcinoma Recurrence in an Ileal Neobladder Nine Years after Primary Surgery with Muir-Torre Syndrome. Case Rep Urol. 2016; 2016: 4762514. doi: 10.1155/2016/4762514

6. Hamid AR, Ridwan FR., Parikesit D, Widia F, Mochtar CA, Umbas R. Meta-Analysis of Neoadjuvant Chemotherapy Compared to Radical Cystectomy Alone in Improving Overall Survival of Muscle-Invasive Bladder Cancer Patients. BMC Urology. 2020; 158: 20(1). doi: 10.1186/ s12894-020-00733-Z

7. Koie T, Ohyama C, Imai A, Hatakeyama S, Yoneyama T, Hashimoto Y. Recurrence Pattern After Neoadjuvant Chemotherapy Compared to Surgery Alone in Patients with Muscle-Invasive Bladder Cancer. Journal of Clinical Oncology. 2016; 34(2): 374. doi: 10.1200/jco.2016.34.2_ suppl.374

8. Koh, DO, Husband JE. Patterns of Recurrence of Bladder Carcinoma Following Radical Cystectomy. Cancer Imaging. 203; 3(2): 96-100. doi: 10.1102/1470-7330.2003.0009

9. Cakmak O, Tarhan H, Celik O, Kucuk U, Ilbey YO. Transitional Cell Carcinoma in Orthotopic Ileal Neobladder. Case Reports in Urology. 2014; 2-14: 1-3. doi: 10.1155/2014/218615

10. Yamashita R, Matsuzaki M, Niwakawa M, Ito I. Bacillus CalmetteGuérin Treatment of Urothelial Carcinoma Arising in The Ileal Neobladder After Radical Cystectomy. Int J Urol. 2014; 21(3): 333-334. doi: 10.1111/iju.12268

11. Herawi M, Leppert JT, Thomas GV, De Kernion JB, Epstein JI. Implants of Noninvasive Papillary Urothelial Carcinoma in Peritoneum And Ileocolonic Neobladder: Support for "Seed and Soil" Hypothesis Of Bladder Recurrence. Urology. 2006; 67(4): 746-750. doi: 10.1016/j. urology.2005.10.023

None. 